\title{
Governor vessel acupuncture for acute ischemic stroke: a systematic review and meta-analysis
}

\author{
Tian-Yu Shao ${ }^{1}$, Min-Rui Ding ${ }^{2}$, Zi-Xiang Ye ${ }^{3}$, Ming-Xia Qian ${ }^{4}$, Xiu Zhou ${ }^{1}$, Zhu-Qing Jin ${ }^{5}$ \\ ${ }^{1}$ The First School of Clinical Medicine, Zhejiang Chinese Medical University, Hangzhou, China; ${ }^{2}$ Department of Neurology, Longhua Hospital, \\ Shanghai University of Traditional Chinese Medicine, Shanghai, China; ${ }^{3}$ Department of Cardiology, Peking University China-Japan Friendship \\ School of Clinical Medicine, Beijing, China; ${ }^{4}$ School of Public Health, Zhejiang Chinese Medical University, Hangzhou, China; ${ }^{5}$ School of Basic \\ Medical Sciences, Zhejiang Chinese Medical University, Hangzhou, China \\ Contributions: (I) Conception and design: TY Shao, MR Ding, ZQ Jin; (II) Administrative support: ZQ Jin; (III) Provision of study materials or \\ patients: TY Shao, MR Ding, ZX Ye; (IV) Collection and assembly of data: TY Shao, MX Qian, X Zhou; (V) Data analysis and interpretation: TY \\ Shao, MR Ding, ZX Ye, ZQ Jin; (VI) Manuscript writing: All authors; (VII) Final approval of manuscript: All authors. \\ Correspondence to: Zhu-Qing Jin. School of Basic Medical Sciences, Zhejiang Chinese Medical University, Hangzhou 310053, China. \\ Email: jinzq@hotmail.com.
}

Background Acute ischemic stroke (AIS) is the major type of stroke, which highly risks human health and life quality. Governor vessel acupuncture (GV Ac) is one specific acupoint selection treatment. This study aimed to systematically evaluate the clinical value of GV Ac in AIS patients.

Methods: Seven electronic databases were searched for all related randomized controlled trials before December 2020. The included studies should meet the following criteria: all target patients were diagnosed as AIS; the experimental group used GV Ac as the only intervention or combined with routine neurology therapy as conventional treatment; the control group received ordinary acupuncture, or the same conventional treatment as the experimental group, or both. Evaluated the quality of all included trials and performed a meta-analysis of the extracted data.

Results: A total of 18 trials were included, involving 1,543 AIS patients. The results showed compared to the conventional treatment, GV Ac combining with conventional therapy resulted in Barthel Index (BI) (MD $=14.16$, 95\% CI: 7.34, 20.79) improvement, $\mathrm{mRS}(\mathrm{MD}=-0.63,95 \% \mathrm{CI}:-0.95,-0.32, \mathrm{P}<0.0001)$ decrease, better National Institute of Health Stroke Scale (NIHSS) scores (MD =-1.18, 95\% CI: -1.52, -0.83), and lower China Stroke Score (CSS)/Modified Edinburgh-Scandinavia Stroke Scale (MESSS) scores (MD $=-3.77,95 \%$ CI: $-4.98,-2.57)$. Furthermore, GV Ac could better improve activities of daily living (ADL) (MD $=8.27,95 \%$ CI: 4.29, 12.26) and neurological deficit scores (NIHSS: MD =-1.32, 95\% CI: $-2.18,-0.47$; CSS/MESSS: MD =-4.63, 95\% CI: $-5.91,-3.35)$, when compared to the ordinary acupuncture.

Discussion: According to the current evidence, GV Ac for AIS's efficacy appears to be better than that of ordinary acupuncture. When combined with conventional treatment, GV Ac may increase the benefit. But limited by the methodological quality of the included studies, more strictly designed large-scale randomized controlled trials are needed.

Trial registration number CRD42020203480.

Keywords: Governor vessel acupuncture; acute ischemic stroke; meta-analysis; systematic review

Submitted Mar 24, 2021. Accepted for publication May 25, 2021.

doi: 10.21037/apm-21-691

View this article at: https://dx.doi.org/10.21037/apm-21-691 


\section{Introduction}

Stroke is a major disease that threatens human health $(1,2)$. Acute ischemic stroke (AIS) is the most common type of stroke, accounting for approximately $71 \%$ of all types of stroke (3). A prospective study of 1977 AIS patients conducted by Jørgensen et al. (4) found that $80 \%$ of patients achieved the best neurological recovery within 4.5 weeks, and the acute phase was the key to treatment (5). Presently, there are many explorations on the treatment of AIS, but all have limitations. Recombinant tissue plasminogen activator (rt-PA) is widely used. Nevertheless, because of the narrow treatment window $(4.5 \mathrm{~h})$, many contraindications, and the risk of intracranial hemorrhage, only $2-5 \%$ of patients have benefited from it (6).

Acupuncture, as one of complementary alternative medicine (CAM), is widely used in China and other east Asian countries for treating stroke over the past 1,000 years. Due to the limitation of conventional neurology treatment, positive views, and the growing availability of acupuncture, more stroke patients turning to acupuncture. One study showed 54\% of 304 stroke-patient respondents had used CAM, among which acupuncture was the most widely used, and $57 \%$ of users felt effective and $84 \%$ considered it relieved the symptoms. (7) World Health Organization has recommended acupuncture for stroke treatment and care (8).

Jin et al. $(9,10)$ in a previous rats experiment found that governor vessel acupuncture (GV Ac) can promote the recovery of cortical somatosensory evoked potential (CSEP) and prevent the cytotoxicity of $\mathrm{NO}$ by inhibiting the excessive expression of $\mathrm{nNOS}$ mRNA and iNOS mRNA. A clinical trial on patients with AIS further confirmed a more obvious reduction in the scope of ischemic injury and better clinical effects in individuals treated by GV Ac when compared to conventional treatment (11). The main trunk of the GV runs along the posterior midline of the body, ascending along the spine, to the dorsal cranial Fengfu (GV 16) into the skull, making it closely related to the brain and spinal cord. Huang Di Nei fing, one of the four classic volumes of traditional Chinese medicine states: "If the disease is in the brain, first treat the governor vessel." This supports the GV plays a pivotal role in the auxiliary treatment of acute cerebrovascular accidents, hemiplegia, spinal cord injury, and other brain diseases (12).

In recent years, an increasing number of clinical trials and basic studies have provided evidence to support the effectiveness of GV Ac in treating AIS (13). One systematic analysis (14) of 54 basic studies showed that acupuncture based on Baihui (GV 20) can improve cerebral infarction and neurological function scores in an AIS animal model, and exhibits a potential neuroprotective effect. As for clinical trials of GV Ac in human AIS patients, there is still a lack of systematic evaluation and no reliable, evidencebased research to provide evidence of its efficacy, which limits the clinical application of GV Ac in the treatment of AIS to a certain extent.

Currently, many systematic reviews have evaluated the efficacy of acupuncture in the treatment of stroke. However, the existing systematic reviews did not distinguish the included subjects between ischemic and hemorrhagic stroke, and acute, chronic, and convalescent stages. Compared to the previous systematic review, this study limits the selection of acupoints. Besides, the main acupoints in this study focus on the $\mathrm{GV}$, and the included subjects are defined as AIS patients. Overall, this study aims to comprehensively evaluate the efficacy of GV Ac for AIS. An evidence-based foundation can be laid for the further clinical application of governor vessel acupuncture in the treatment of AIS.

We present the following article in accordance with the PRISMA reporting checklist (available at https://dx.doi. org/10.21037/apm-21-691).

\section{Methods}

It was registered on PROSPERO (https://www.crd.york. ac.uk/PROSPERO/; trial registration CRD42020203480).

\section{Search strategy}

We searched China National Knowledge Infrastructure (CNKI, https://www.cnki.net/), VIP Journal Database (http://www.cqvip.com/), Wanfang Database (http:// www.wanfangdata.com.cn), Chinese Biomedical Database (CBM, http://www.sinomed.ac.cn/), Cochrane Library, Web of Science and PubMed. A comprehensive search was conducted on clinical studies of GV Ac for AIS before December 2020. The keywords used were as follows: (Brain Infarction OR Cerebral Infarction OR Ischemic Stroke OR Cerebral Infarction OR Ischemia Apoplexy OR Ischemic Apoplexy OR Brain Ischemia OR Brain Ischemias OR Ischemia, Brain OR Ischemic Encephalopathy OR Encephalopathy, Ischemic OR Ischemic Encephalopathies OR Cerebral Ischemia OR Cerebral Ischemias OR Ischemias, Cerebral OR Ischemia, Cerebral) AND (governor vessel OR du mai OR governor meridian OR du meridian) AND acupuncture. At the same time, 
the included literature's references were reviewed. The completed but unpublished clinical studies were searched through the National Institute of Health Clinical Trials Database (http://clinicaltrials.gov) and Chinese Clinical Trial Registry (www.chictr.org.cn/searchproj.asp) to find any relevant studies that may have been missed.

\section{Eligibility criteria}

Randomized controlled trials were included. All patients had an AIS diagnosis according to the following criteria: (I) diagnosed by head CT, MRI, or according to the AIS diagnosis found in "Chinese guidelines for diagnosis and treatment of acute ischemic stroke"; (II) patients were within 30 days of onset (15). The types of interventions are as follow: (I) In the experimental group, the main acupoints were on the GV, which could be the only intervention or combined with the conventional treatments. The conventional treatments include thrombolysis or defibrination, anticoagulant, antiplatelet aggregation, cerebral metabolism protection, cerebral edema reduction, cerebral circulation improvement, intracranial pressure reduction, and other symptomatic, supportive and prevention therapy of complications, can also combine with traditional Chinese medicine, moxibustion, or rehabilitation training. (II) The control group received ordinary acupuncture, which was adjusted according to "Acupuncture and moxibustion therapy" (16) or only received the same conventional treatment as the experimental group; or both. Other exclusion criteria: repeated publication of the same or incomplete data; or the curative effect evaluation criteria were not clear.

\section{Types of outcome measures}

Activities of daily living (ADL): (I) Barthel Index (BI) (II) the Modified Rankin Scale (mRS) is a 7-level ordinal score (graded from 0 to 6, 6 for death). All three studies that included this measure were analyzed as continuous.

Neurological function score: (I) National Institute of Health Stroke Scale (NIHSS) (II) China Stroke Score (CSS) was revised based on Modified Edinburgh-Scandinavia Stroke Scale (MESSS) (17). In this study, CSS and MESSS scores were combined for analysis $(14,17)$.

\section{Data extraction and quality evaluation}

Eligible studies were assessed independently by 2 authors
(Shao and Qian), and data were extracted using prespecified forms. The quality of the studies using the Cochrane risk of bias tool (18), Any difference was resolved through discussion with the third senior researcher (Jin).

\section{Data analysis and statistical methods}

The statistical software Revman 5.3 and Stata 13 provided by the International Evidence-based Medicine Collaboration Network were used to analyze the collected data. Dichotomous outcomes were expressed as a ratio (OR), and the continuous outcomes were expressed by the mean difference (MD) or standardized mean difference (SMD), both of which are expressed by $95 \%$ confidence intervals (CI). Heterogeneity was assumed when $\mathrm{P}<0.05$ or $\mathrm{I}^{2}>50 \%$. And subgroup analysis was performed when heterogeneity was high. The final results were described by the forest map. Publication bias was analyzed by the Egger test. If $\mathrm{P}>0.05$, no publication bias was detected.

\section{Results}

A total of 186 related articles were retrieved. According to the eligible criteria, 18 articles were included. A flow chart of data acquisition is shown in Figure 1.

\section{Study characteristics}

All 18 included studies were from China, with 1,543 participants. The sample size varied from 37 to 256 patients. Except for Jia et al. (19) was a three-arm study, the other seventeen studies were all double-arm studies. All studies reported baseline similarity of patients before treatment. Seven studies set up ordinary acupuncture as a control, and 12 studies used conventional treatment as control. Conventional treatment included therapies recommended by the "Guidelines for early endovascular treatment of acute ischemic stroke" (20) from the American Stroke Association and the "Chinese guidelines for diagnosis and treatment of acute ischemic stroke" (15). The characteristics of the included studies are shown in Table 1.

\section{Bias and methodological quality}

The Cochrane risk of bias tool was used to evaluate bias risk and the quality of the included studies. Figure 2 lists the specific evaluation details of each study. All the studies reported randomization, but seven of which did not describe the random sequence generation methods clearly. Two studies mentioned allocation concealment $(23,26)$ 


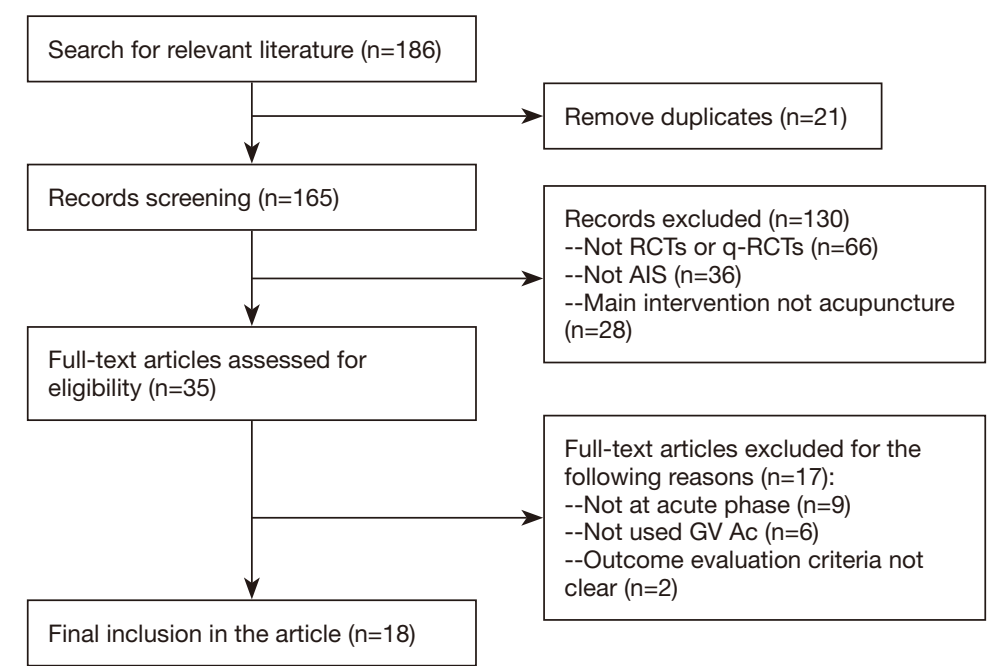

Figure 1 Flowchart of literature acquisition. RCT, randomized controlled trial; AIS, Acute ischemic stroke; GV Ac, governor vessel acupuncture.

Table 1 Characteristics of included studies

\begin{tabular}{|c|c|c|c|c|c|}
\hline \multirow{2}{*}{ Included trials } & \multirow{2}{*}{$\begin{array}{l}\text { Disease } \\
\text { duration } \\
\text { before } \\
\text { treatment }\end{array}$} & \multicolumn{2}{|c|}{$\begin{array}{l}\text { No. of participants (male/female); } \\
\text { age (years; mean } \pm \text { SD) }\end{array}$} & \multicolumn{2}{|l|}{ Intervention } \\
\hline & & GV Ac & Control & GV Ac & Control \\
\hline Liu 2020, (21) & $6-33 \mathrm{~h}$ & 63 & 63 & $\begin{array}{l}\text { Conventional treatment + GV Ac GV20, GV26, } \\
\text { RN23, RN4, RN6, RN12 }\end{array}$ & $\begin{array}{c}\text { Conventional } \\
\text { treatment }+\mathrm{Ac}\end{array}$ \\
\hline $\begin{array}{l}\text { Guan } \\
2019,(22)\end{array}$ & $<3 d$ & $\begin{array}{c}30(16 / 14) \\
(70.57 \pm 14.73)\end{array}$ & $\begin{array}{c}30(16 / 14) \\
(68.33 \pm 11.09)\end{array}$ & $\begin{array}{l}\text { Conventional treatment + GV Ac GV20, GV16, } \\
\text { GV24, GB20, PC6, LI4, ST36, SP6 }\end{array}$ & $\begin{array}{l}\text { Conventional } \\
\text { treatment }\end{array}$ \\
\hline Lai 2019, (23) & $<3 d$ & $\begin{array}{c}37(21 / 16) \\
(65.58 \pm 8.57)\end{array}$ & $\begin{array}{c}36(19 / 17) \\
(67.37 \pm 10.46)\end{array}$ & $\begin{array}{l}\text { Conventional treatment + GV Ac GV20, GV26, } \\
\text { GV14, GV16, RN6, RN4, GV24, EX-HN3 }\end{array}$ & $\begin{array}{l}\text { Conventional } \\
\text { treatment }\end{array}$ \\
\hline Xu 2019, (24) & $<1 \mathrm{~m}$ & $\begin{array}{c}46(27 / 19) ; 43-76 \\
(59.92 \pm 7.26)\end{array}$ & $\begin{array}{c}46(25 / 21) ; 42-78 \\
(60.17 \pm 7.39)\end{array}$ & $\begin{array}{l}\text { Conventional treatment + GV Ac GV20, GV14, } \\
\text { GB20, LI11, LI4, ST36, SP6 }\end{array}$ & $\begin{array}{l}\text { Conventional } \\
\text { treatment }\end{array}$ \\
\hline $\begin{array}{l}\text { Hua } \\
2019,(25)\end{array}$ & $24 \mathrm{~h}-2 \mathrm{w}$ & 50 (29/21); $(64 \pm 9)$ & $\begin{array}{l}50(31 / 19) \\
\quad(62 \pm 12)\end{array}$ & $\begin{array}{l}\text { Conventional treatment + GV Ac GV20, GV14, } \\
\text { GB20, LI11, LI4, ST36, SP6 }\end{array}$ & $\begin{array}{l}\text { Conventional } \\
\text { treatment }\end{array}$ \\
\hline Li 2018, (27) & $<24 \mathrm{~h}$ & $\begin{array}{c}18(11 / 7) \\
(58.1 \pm 8.69)\end{array}$ & $\begin{array}{c}19(10 / 9) \\
(57.8 \pm 8.55)\end{array}$ & $\begin{array}{l}\text { Conventional treatment + GV Ac GV20, GV4, GV26, } \\
\text { GV23, GV15, GV16, GV8, GV3, EX-B2 }\end{array}$ & $\begin{array}{l}\text { Conventional } \\
\text { treatment }\end{array}$ \\
\hline Xu 2018, (28) & $3-7 d$ & $\begin{array}{c}28(16 / 12) \\
47-80(63 \pm 8)\end{array}$ & $\begin{array}{c}27(12 / 15) ; 52-78 \\
(64 \pm 8)\end{array}$ & $\begin{array}{l}\text { Conventional treatment + GV Ac GV20, GV4, GB20, } \\
\text { GV18, GV24, GV26, EX-HN3, LI11, LI4, ST36 }\end{array}$ & $\begin{array}{l}\text { Conventional } \\
\text { treatment }+\mathrm{Ac}\end{array}$ \\
\hline Liu 2017, (29) & $<48 \mathrm{~h}$ & 40 (20/20); 53-72 & 38 (20/18); 55-70 & GV Ac GV20, GV26, RN23, RN4, RN6, RN12 & $A c$ \\
\hline $\begin{array}{l}\text { Luan } \\
2017,(30)\end{array}$ & $14 \mathrm{~d}$ & $\begin{array}{c}43(22 / 21) \\
(65.39 \pm 4.85)\end{array}$ & $\begin{array}{c}43(23 / 20) \\
(64.39 \pm 3.95)\end{array}$ & $\begin{array}{l}\text { Conventional treatment + GV Ac GV20, GV26, RN4, } \\
\text { RN12 }\end{array}$ & $\begin{array}{l}\text { Conventional } \\
\text { treatment }\end{array}$ \\
\hline $\begin{array}{l}\text { Zhang } \\
\text { LH 2017, (31) }\end{array}$ & $<1 \mathrm{~m}$ & $\begin{array}{c}32(18 / 14) \\
(66.53 \pm 8.79)\end{array}$ & $\begin{array}{c}32(17 / 15) \\
(59.84 \pm 7.75)\end{array}$ & $\begin{array}{l}\text { Conventional treatment + GV Ac GV20, GV4, GV16, } \\
\text { GV24, GV4 }\end{array}$ & $\begin{array}{l}\text { Conventional } \\
\text { treatment } \\
\quad+\mathrm{Ac}\end{array}$ \\
\hline
\end{tabular}

Table 1 (continued) 
Table 1 (continued)

\begin{tabular}{|c|c|c|c|c|c|}
\hline \multirow{2}{*}{ Included trials } & \multirow{2}{*}{$\begin{array}{l}\text { Disease } \\
\text { duration } \\
\text { before } \\
\text { treatment }\end{array}$} & \multicolumn{2}{|c|}{$\begin{array}{l}\text { No. of participants (male/female); } \\
\text { age (years; mean } \pm \text { SD) }\end{array}$} & \multicolumn{2}{|l|}{ Intervention } \\
\hline & & GV Ac & Control & GV Ac & Control \\
\hline Cai 2016, (33) & $4-5 \mathrm{~h}$ & $\begin{array}{l}43(30 / 13) ; 46-78 \\
\quad(57.2 \pm 6.4)\end{array}$ & $\begin{array}{c}30(15 / 15) ; 38-72 \\
(54.01 \pm 11.72)\end{array}$ & $\begin{array}{l}\text { Conventional treatment + GV Ac GV20, GV14, } \\
\text { GV26, GV4, GV9 }\end{array}$ & $\begin{array}{l}\text { Conventional } \\
\text { treatment }\end{array}$ \\
\hline Ma 2016, (34) & $<48 \mathrm{~h}$ & $\begin{array}{c}40(20 / 20) \\
(62.28 \pm 4.38)\end{array}$ & $\begin{array}{c}38(20 / 18) \\
(61.26 \pm 4.44)\end{array}$ & GV Ac GV20, GV26, RN23, RN4, RN6, RN12 & Ac \\
\hline Li 2009, (35) & $3 \mathrm{~h}-11 \mathrm{~d}$ & $\begin{array}{c}132(76 / 56) ; 43-76 \\
(66.2)\end{array}$ & $\begin{array}{l}124(70 / 54) \\
47-78(67.9)\end{array}$ & $\begin{array}{l}\text { Conventional treatment + GV Ac GV20, GV14, } \\
\text { GV26, GV16, GV13, GV12, GV11, GV9, GV8, GV6, } \\
\text { GV5, GV4 }\end{array}$ & $\begin{array}{l}\text { Conventional } \\
\text { treatment }\end{array}$ \\
\hline Jia 2009, (19) & $<5 d$ & $\begin{array}{c}24(14 / 10) \\
(65.92 \pm 11.77)\end{array}$ & $\begin{array}{l}\text { Conventional } \\
\text { treatment: } \\
23(13 / 10), \\
(67.30 \pm 9.29) ; \\
\text { Ac: } 21(12 / 9) \\
(66.24 \pm 11.64)\end{array}$ & $\begin{array}{l}\text { Conventional treatment + GV Ac GV20, GV14, } \\
\text { GV16, GV26, LI15, LI11, SJ5, LI4, ST34, ST36, } \\
\text { ST40, SP6, LV3 }\end{array}$ & $\begin{array}{c}\text { Conventional } \\
\text { treatment/ } \\
\text { conventional } \\
\text { treatment + Ac }\end{array}$ \\
\hline $\begin{array}{l}\text { Zhang } \\
\text { SJ 2007, (36) }\end{array}$ & $<5 d$ & $\begin{array}{l}40(29 / 11) \\
(61.3 \pm 11.4)\end{array}$ & $\begin{array}{l}40(27 / 13) \\
(62.4 \pm 10.9)\end{array}$ & $\begin{array}{l}\text { Conventional treatment + GV Ac GV20, GV4, GV26, } \\
\text { GV16 }\end{array}$ & $\begin{array}{l}\text { Conventional } \\
\text { treatment }\end{array}$ \\
\hline Jin 1999, (11) & $<1 \mathrm{~m}$ & 60 & 60 & $\begin{array}{l}\text { Conventional treatment + GV Ac GV20, GV26, } \\
\text { GV23 }\end{array}$ & $\begin{array}{l}\text { Conventional } \\
\text { treatment }\end{array}$ \\
\hline
\end{tabular}

GV Ac, governor vessel acupuncture.

and Lai et al. (23) mentioned blindness. But because of the particularity of acupuncture, patients and acupuncture operators could not be blinded without a sham acupuncture intervention in the control group; therefore these studies have an inherently high risk of performance bias. Two studies [Xu et al. (28), Zhang L et al. (32)] reported case loss with a small loss rate (Xu 5/60, Zhang 3/40), and none of the other studies reported case loss, so the incomplete outcome data were assessed as low bias risk.

\section{ADL of governor vessel acupuncture + conventional therapy versus conventional therapy}

\section{Activities of daily living (ADL)}

A total of eight articles reported the effect of GV Ac combined with conventional therapy compared with conventional therapy on ADL, which was evaluated by $\mathrm{BI}$ and mRS. Five studies used BI as an evaluation (MD $=14.16,95 \%$ CI: $7.34,20.79, \mathrm{P}<0.0001)$ and duration of the treatment was performed for subgroup analysis (Figure $3 A$ ). Three studies were evaluated by the mRS (MD $=-0.63,95 \%$ CI: $-0.95,-0.32, \mathrm{P}<0.0001$, Figure $3 B)$. The results supported that GV Ac combined with conventional therapy can better improve the ADL of patients.

\section{Neurological deficit score}

A total of six studies reported the effect of GV Ac combined with conventional therapy compared with conventional therapy on the neurological deficit score of patients. Four studies used NIHSS (MD $=-1.18,95 \%$ CI: $-1.52,-0.83$, $\mathrm{P}<0.00001$, Figure 3C) and three studies used CSS/MESSS $(\mathrm{MD}=-3.77,95 \% \mathrm{CI}:-4.98,-2.57, \mathrm{P}<0.00001$, Figure $3 D)$. The results show GV Ac combined with conventional therapy showed a consistent effect on decreasing the patients' neurological impairment.

\section{Governor vessel acupuncture versus ordinary acupuncture}

\section{Activities of daily living (ADL)}

A total of five articles reported the ADL recovery of patients treated with GV Ac compared to ordinary acupuncture, as evaluated by BI. The results showed that the BI score in the GV Ac group was higher than that in the Ac group ( $\mathrm{MD}=8.27,95 \% \mathrm{CI}: 4.29,12.26, \mathrm{P}<0.0001)$. Because of the high heterogeneity test results $(\mathrm{P}=0.001, \mathrm{I} 2$ $=78 \%$ ), we performed subgroup analysis according to the stroke severity at baseline (Figure $4 A$ ) and whether use the electropuncture (Figure 4B). The results support that GV Ac can improve the ADL of patients better than ordinary 


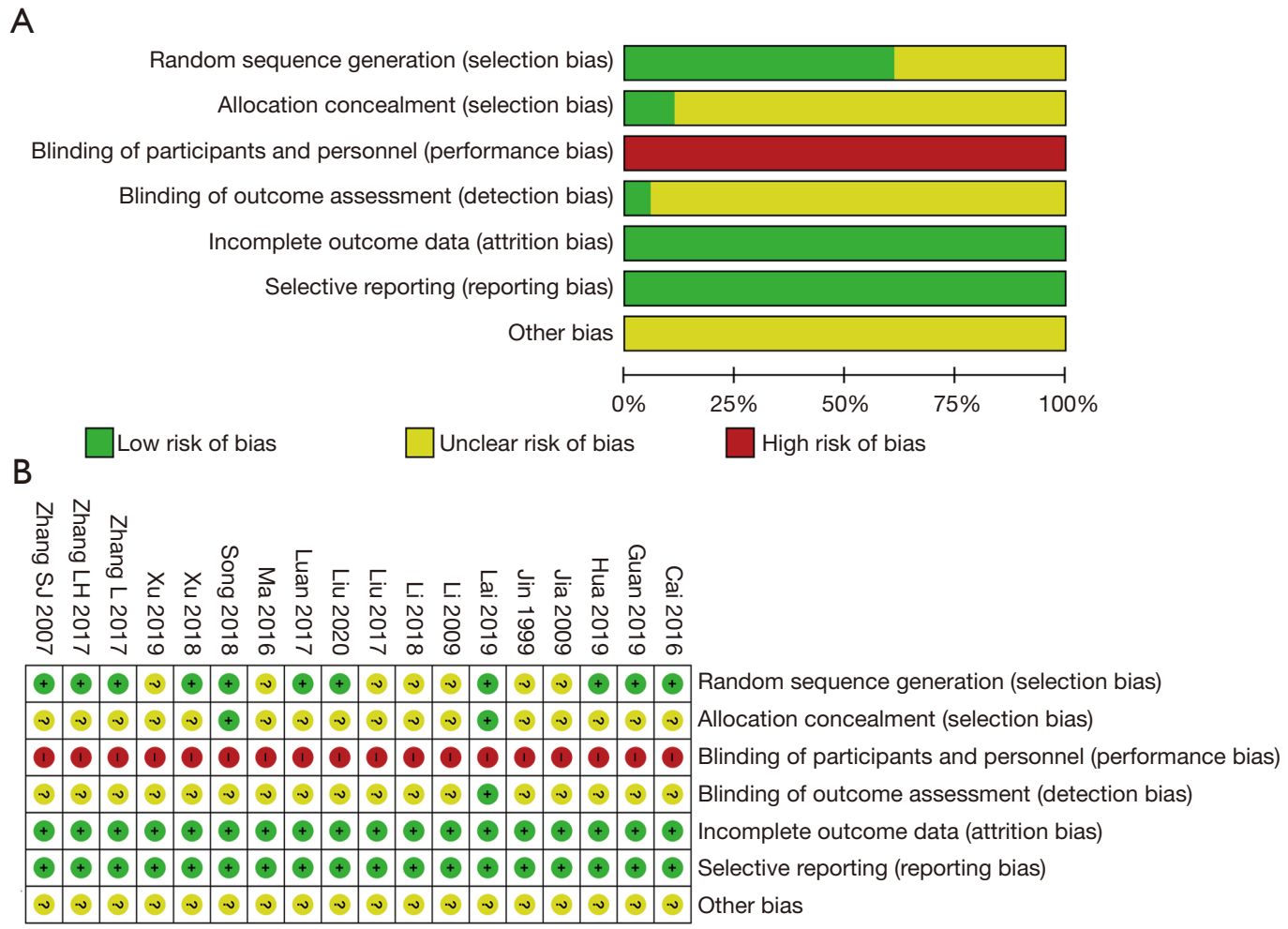

Figure 2 The bias risk and the quality evaluation results of each included study by the Cochrane risk of bias tool. (A) Risk of bias graph. (B) Risk of bias summary.

acupuncture.

\section{Neurological deficit score}

A total of five studies reported the effect of GV Ac on neurological deficit score compared with ordinary acupuncture. Two studies used NIHSS (MD $=-1.32,95 \%$ CI: $-2.18,-0.47, \mathrm{P}=0.002$, Figure $4 C$ ). Three studies used CSS/MESSS to evaluate the neurological deficit score $(\mathrm{MD}=-4.63,95 \% \mathrm{CI}:-5.91,-3.35, \mathrm{P}<0.00001)$ and we performed subgroup analysis (Figure $4 D$ ). The results supported GV Ac can better improve the neurological impairment of the patients.

\section{Adverse reaction}

A total of three studies $(23,26,27)$ reported the occurrence of adverse events during treatment, while the other 15 studies did not mention adverse reactions. There was one case of subcutaneous hematoma and needle stagnation in GV Ac and ordinary acupuncture, respectively, in Song (26).
There was no significant difference in routine laboratory tests before and after treatment in $\mathrm{Li}$ (27), and three cases of subcutaneous hematoma appeared in the GV Ac group. Lai et al. (23) reported occasional acupuncture point bleeding. No other major adverse reactions were found.

\section{Publication bias}

Egger linear regression is recommended to determine publication bias analysis in the latest Cochrane manual. $\mathrm{BI}$ is the main outcome index, so the publication bias is evaluated by the funnel chart. The results showed that the intercept line crossed zero, $\mathrm{P}=0.171>0.05$, indicating no obvious publication bias (Figure 5).

\section{Discussion}

This meta-analysis included 18 studies, with a total of 1,543 patients. Compared with a previous systematic review, this study only included the patients who were 


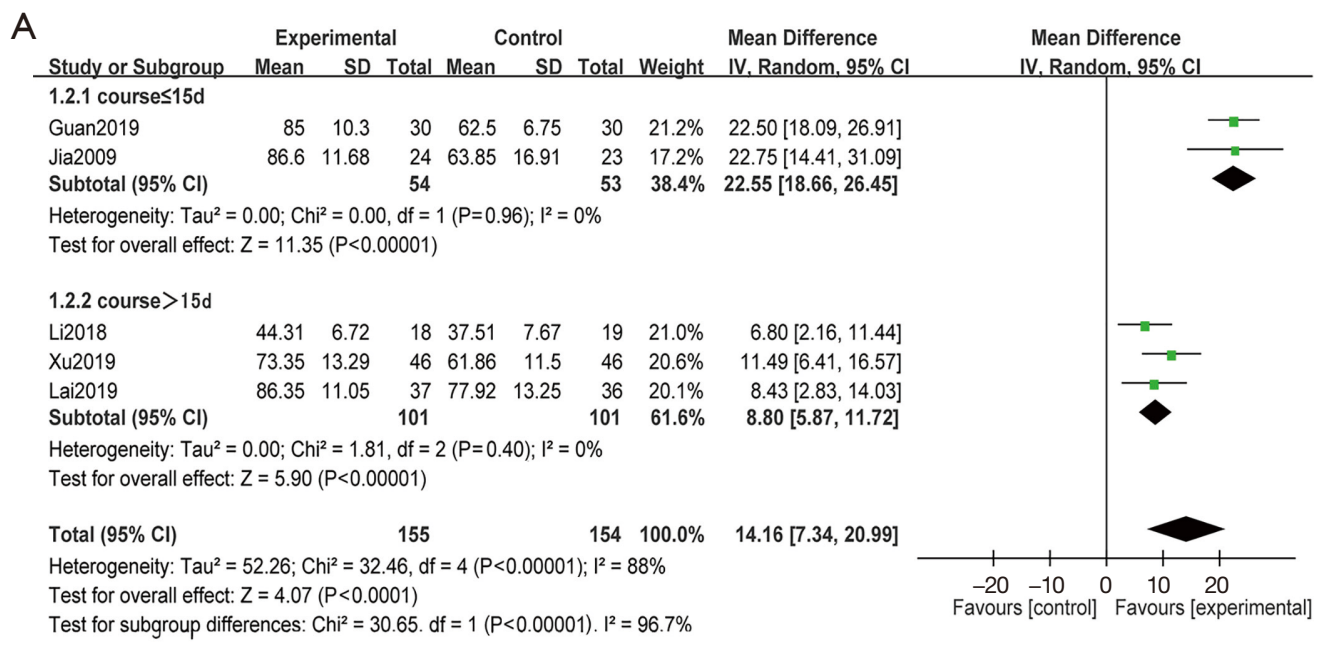

B

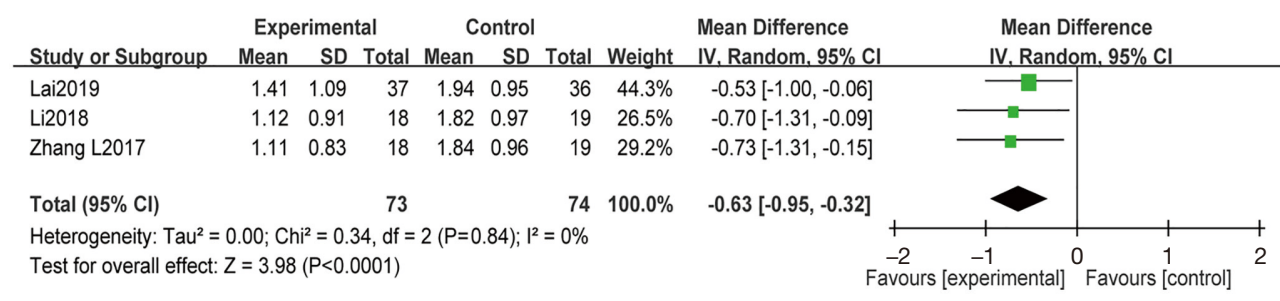

C

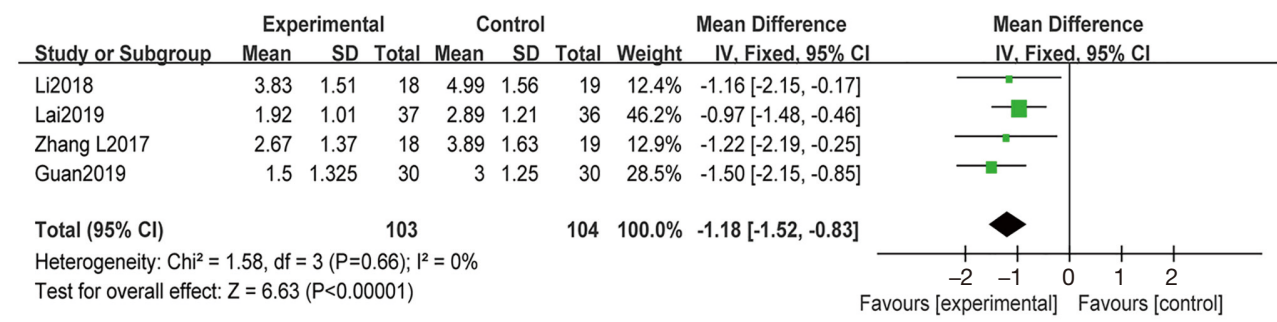

D

\begin{tabular}{|c|c|c|c|c|c|c|c|c|c|}
\hline Study or Subgroup & \multicolumn{3}{|c|}{ Experimental } & \multicolumn{2}{|c|}{ Control } & Total & \multicolumn{2}{|c|}{$\begin{array}{cc} & \text { Mean Difference } \\
\text { Weight } & \text { IV. Fixed, } 95 \% \mathrm{Cl}\end{array}$} & $\begin{array}{l}\text { Mean Difference } \\
\text { IV. Fixed, } 95 \% \mathrm{CI}\end{array}$ \\
\hline Cai2016 & 14.36 & 2.39 & 30 & 18.08 & 3.65 & 30 & $60.0 \%$ & $-3.72[-5.28,-2.16]$ & \\
\hline $\mathrm{Jia} 2009$ & 7.17 & 5.03 & 24 & 10.3 & 4.73 & 23 & $18.8 \%$ & $-3.13[-5.92,-0.34]$ & \\
\hline Zhang SJ2007 & 10.38 & 3.49 & 40 & 14.88 & 7.71 & 40 & $21.3 \%$ & $-4.50[-7.12,-1.88]$ & \\
\hline Total $(95 \% \mathrm{Cl})$ & & & 94 & & & 93 & $100.0 \%$ & $-3.77[-4.98,-2.57]$ & \\
\hline $\begin{array}{l}\text { Heterogeneity: } \mathrm{Chi}^{2}= \\
\text { Test for overall effect: }\end{array}$ & $\begin{array}{l}.50, d f= \\
Z=6.12\end{array}$ & $\begin{array}{l}=2(P=C \\
(P<0.0\end{array}$ & $\begin{array}{l}0.78) ; \\
0001)\end{array}$ & $\left.\right|^{2}=0 \%$ & & & & & $\begin{array}{cccc}-4 & -2 & 0 & 2 \\
\text { xperimental] } & \text { Favours }\end{array}$ \\
\hline
\end{tabular}

Figure 3 The forest plot and subgroup analysis results of ADL and neurological deficit score comparing GV Ac combined with conventional therapy with conventional therapy. (A) Forest plot and subgroup analysis of BI. (B) Forest plot of mRS. (C) Forest plot of NIHSS. (D) Forest plot of CSS/MESSS. ADL, activities of daily living; GV Ac, governor vessel acupuncture; BI, Barthel Index; mRS, Modified Rankin Scale; NIHSS, National Institute of Health Stroke Scale; CSS, China Stroke Score; MESSS, Modified Edinburgh-Scandinavia Stroke Scale. 


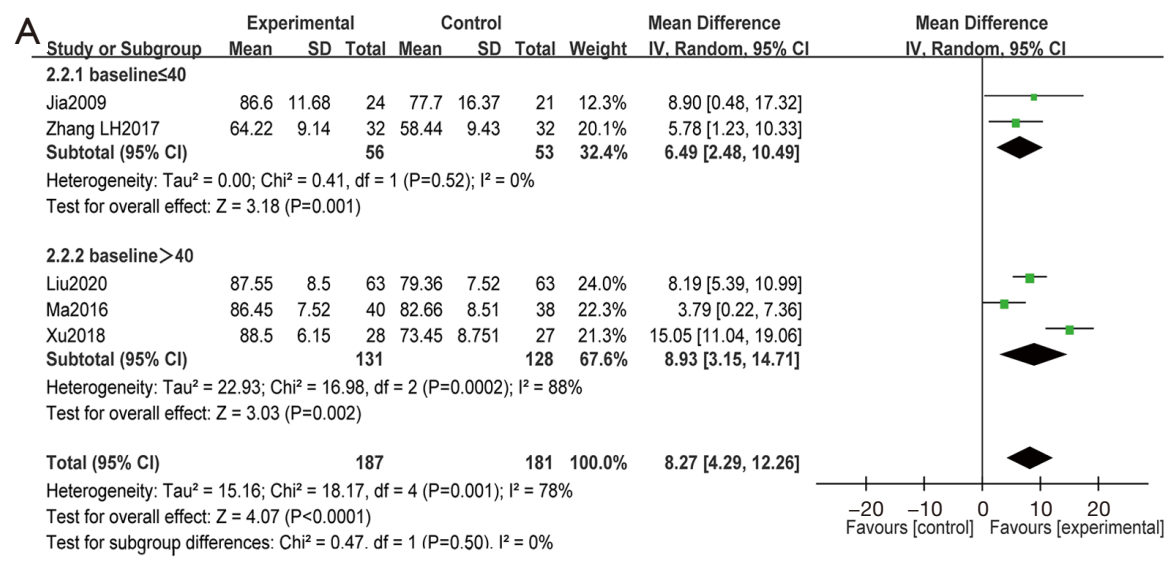

B

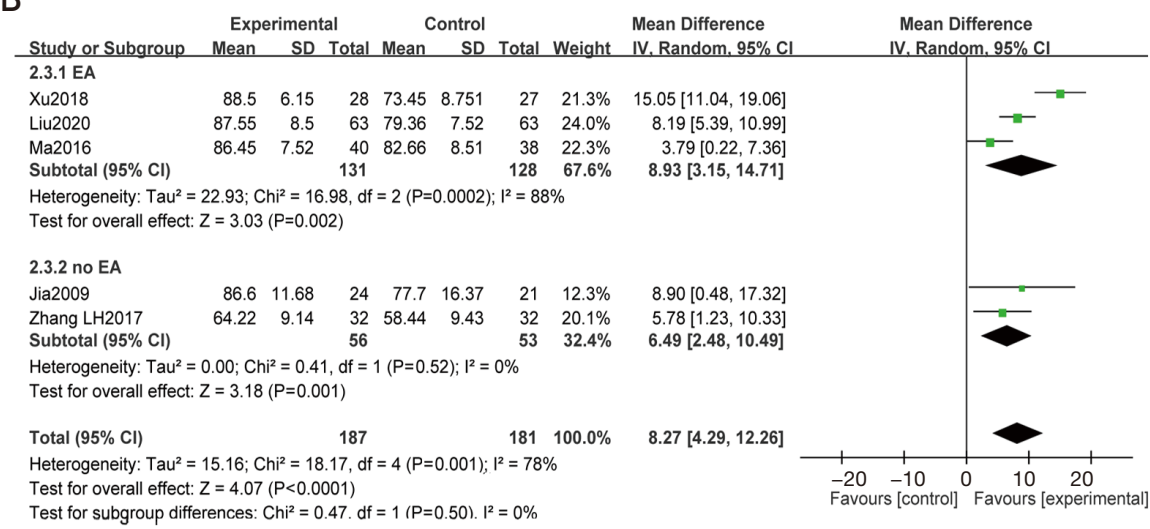

C

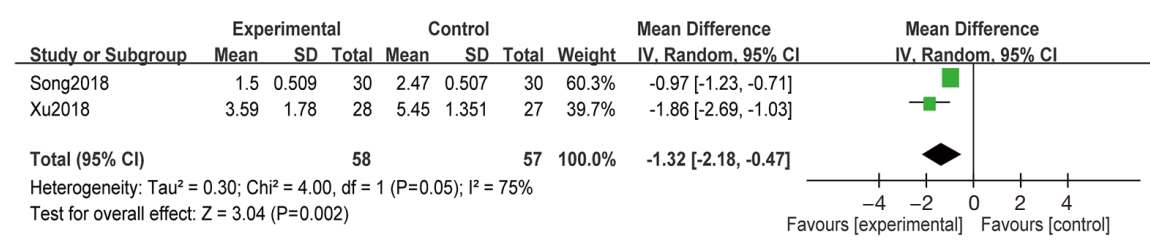

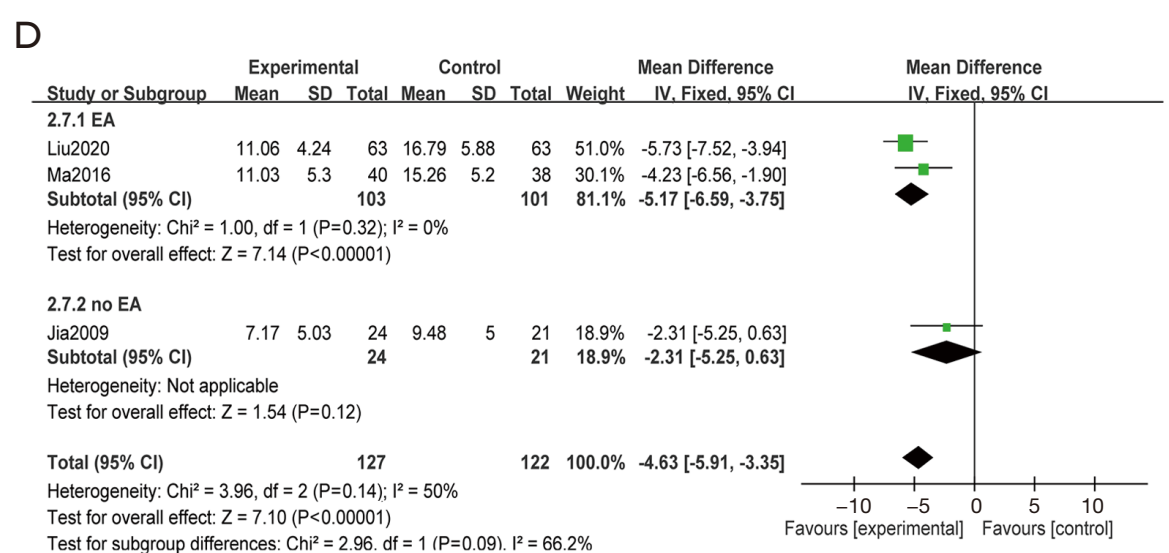

Figure 4 The forest plot and subgroup analysis results of ADL and neurological deficit score comparing GV Ac with ordinary acupuncture. (A) Subgroup analysis of BI according to the baseline stroke severity. (B) Subgroup analysis of BI according to whether use the electropuncture. (C) Forest plot of NIHSS. (D) Forest plot and subgroup analysis of CSS/MESSS. ADL, activities of daily living; GV Ac, governor vessel acupuncture; BI, Barthel Index; NIHSS, National Institute of Health Stroke Scale; CSS, China Stroke Score; MESSS, Modified Edinburgh-Scandinavia Stroke Scale. 


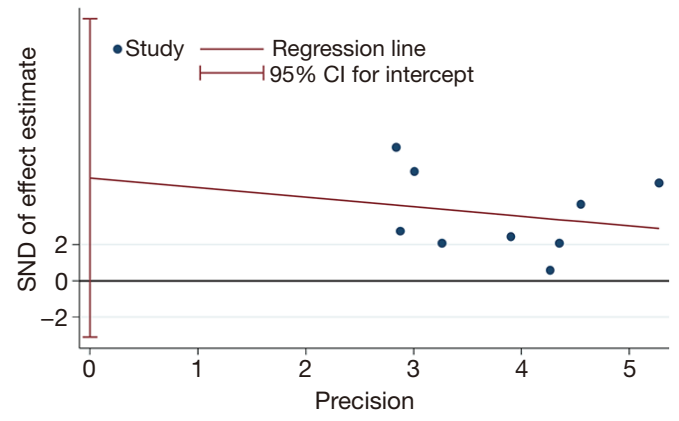

Figure 5 Publication bias analysis.

strictly limited to the acute stage of ischemic stroke and limited the main acupoint to the governor vessel. It's the first systematic analysis to focus on the specific acupoints selection in treating AIS. We systematically evaluated GV Ac for AIS from the aspects of ADL, neurological function score, and adverse reactions. The results were as follows: (I) Among the 18 studies, a total of 12 studies compared GV Ac plus conventional treatment with conventional treatment alone. The results showed that, compared with conventional treatment, GV Ac could better improve the ADL and neurological deficit score; (II) among the 18 studies, a total of seven studies compared GV Ac and ordinary acupuncture. The results showed that the former demonstrated better effects in improving patients' BI, NIHSS, and CSS/MESSS score, may have a better efficacy on the recovery of patients' neurological function; (III) from the existing evidence, GV Ac for AIS seems to be safe, with no major adverse reactions.

In this systematic review, we found for treating AIS the most commonly used GV acupoints were Baihui (GV 20), Renzhong (GV 26), Dazhui (GV 14), and Fengfu (GV 16). Some relevant laboratory studies shed the light on the possible physiologic mechanisms. Jin et al. (10) explored the effects of GV Ac on transient focal cerebral ischemia rats and found GV Ac could promote the recovery of cortical somatosensory evoked potentials and reduce the volume of cerebral infarction after cerebral ischemia, and may stronger than ordinary acupuncture. Another study from Jin et al. (37) showed the levels of MCPIP1 protein and mRNA in the brain of middle cerebral artery occlusion (MCAO) mice treated with electroacupuncture Baihui (GV 20) were significantly higher than nonelectroacupuncture group mice, and the activation of NF- $\mathrm{KB}$ signal pathway decreased, while MCPIP1 deficient mice could not inhibit NF- $\kappa \mathrm{B}$ signal pathway. Therefore, it was speculated MCPIP1 may participate in delayed cerebral ischemic tolerance by inhibiting NF- $\mathrm{KB}$ signal pathway when electroacupuncture Baihui (GV 20). Wang et al. (38) have also found electroacupuncture Baihui (GV 20) could activate the anti-apoptotic cannabinoid receptor mediated by endogenous epsilon protein kinase $\mathrm{C}(\varepsilon \mathrm{PKC})$ in rats with middle cerebral artery occlusion, thus protecting the ischemic injury after focal cerebral ischemia. Tian et al. (39) used protein-chip to explore the mechanism of acupuncture at Baihui (GV 20), Renzhong (GV 26), and Dazhui (GV 14) on the middle cerebral artery occlusion rat model. It was found the number of activated proteins in the GV Ac group was not only higher than that in the acupuncture control group but also different. Most of the activated proteins in the GV Ac group were key proteins of the signal pathway, indicating that GV Ac could activate a variety of signal pathways and promote the recovery of brain tissue after ischemic injury.

However, this study also has some limitations. First, the included clinical trials are all conducted in China, so the results may not generalize to other races. In the aspect of blind design, only one study reported single-blind (blind to evaluators). The double-blind trial of acupuncture research is still controversial. Sham-acupuncture may have potential physiological effects (40). Furthermore, it can be complicated to conduct completely blind in some countries, such as China, because patients may be very familiar with the feeling of "de qi." (41). Additionally, not all patients are willing to receive sham acupuncture. In this case, future clinical studies should use blind methods in the random allocation and result in the evaluation as far as possible.

\section{Conclusions}

In summary, there is evidence that GV Ac is more effective than ordinary acupuncture for AIS. Clinical combined with conventional treatment can increase the benefit to patients. As the first summary of GV Ac for AIS, this study may provide guidelines and evidence-based for clinical accurate acupuncture. However, more high-quality, large-sample, strictly designed, randomized controlled trials in China and abroad are needed in the future to confirm these conclusions further.

\section{Acknowledgments}

Funding: This work was supported by the National Natural Science Foundation of China [81774010]; and the Zhejiang Natural Science Foundation Project [ly14h270013]. 


\section{Footnote}

Reporting Checklist: The authors have completed the PRISMA reporting checklist. Available at https://dx.doi. org/10.21037/apm-21-691

Conflicts of Interest: All authors have completed the ICMJE uniform disclosure form (available at https://dx.doi. org/10.21037/apm-21-691). The authors have no conflicts of interest to declare.

Ethical Statement: The authors are accountable for all aspects of the work in ensuring that questions related to the accuracy or integrity of any part of the work are appropriately investigated and resolved.

Open Access Statement: This is an Open Access article distributed in accordance with the Creative Commons Attribution-NonCommercial-NoDerivs 4.0 International License (CC BY-NC-ND 4.0), which permits the noncommercial replication and distribution of the article with the strict proviso that no changes or edits are made and the original work is properly cited (including links to both the formal publication through the relevant DOI and the license). See: https://creativecommons.org/licenses/by-nc-nd/4.0/.

\section{References}

1. Wang L, Liu J, Yang Y, et al. The prevention and treatment of stroke in China still faces great challenges -- Summary of China Stroke Prevention and Treatment Report 2018. China Circulation Journal 2019;34:105-19.

2. Lindsay MP, Norrving B, Sacco RL, et al. World Stroke Organization (WSO): Global Stroke Fact Sheet 2019. Int J Stroke 2019;14:806-17.

3. Campbell BCV, De Silva DA, Macleod MR, et al. Ischaemic stroke. Nat Rev Dis Primers 2019;5:70.

4. Jørgensen HS, Nakayama H, Raaschou HO, et al. Outcome and time course of recovery in stroke. Part II: Time course of recovery. The Copenhagen Stroke Study. Arch Phys Med Rehabil 1995;76:406-12.

5. Lackland DT, Roccella EJ, Deutsch AF, et al. Factors influencing the decline in stroke mortality: a statement from the American Heart Association/American Stroke Association. Stroke 2014;45:315-53.

6. Wan JJ, Wang PY, Zhang Y, et al. Role of acute-phase protein ORM in a mice model of ischemic stroke. J Cell Physiol 2019;234:20533-45.
7. Shin YI, Yang CY, Joo MC, et al. Patterns of using complementary and alternative medicine by stroke patients at two university hospitals in Korea. Evid Based Complement Alternat Med 2008;5:231-5.

8. Zhang S, Li N, Liu M. Use of acupuncture for stroke in China. Acupunct Med 2009;27:146.

9. Jin Z, Cheng J. Effects of electricity on nitric oxide synthase expression in the brain of middle cerebral artery occlusion rats. Acupuncture Research 1998;(6):3-5.

10. Jin Z, Cheng J. Effects of acupuncture at different points on cortical somatosensory evoked potential and cerebral infarction volume in rats with transient focal cerebral ischemia. Shanghai Journal of Acupuncture and Moxibustion 1998;(5):3-5.

11. Jin Z, Gu F, Chen R, et al. The therapeutic effect of Dumai acupoint acupuncture on patients with acute cerebral infarction. Acupuncture Research 1999;(1):3-5.

12. Kim YI, Kim SS, Sin RS, et al. Study on the Cerebral Blood Flow Regulatory Features of Acupuncture at Acupoints of the Governor Vessel. Med Acupunct 2018;30:192-7.

13. Wang WW, Xie CL, Lu L, et al. A systematic review and meta-analysis of Baihui (GV20)-based scalp acupuncture in experimental ischemic stroke. Sci Rep 2014;4:3981.

14. Criteria for diagnosis and Efficacy evaluation of Apoplexy (trial). Journal of Beijing University of Traditional Chinese Medicine 1996;19:55-6.

15. Peng B, Wu B. Chinese Guidelines for the Diagnosis and Treatment of Acute ischemic Stroke 2018. Chinese Journal of Neurology 2018;51:666-82.

16. Liang F. Acupuncture and moxibustion therapy. 2012.

17. Criteria for the degree of clinical Neurological Impairment in stroke patients (1995). Chinese Journal of Neurology 1996;6:62-4.

18. Higgins J, Green SJCdosr. Cochrane Handbook for Systematic Reviews of Interventions. 2011.

19. Jia C. Observation of clinical effect of Du-meridian acupuncture on acute cerebral infarction: Nanjing University of Traditional Chinese Medicine; 2009.

20. Powers WJ, Rabinstein AA, Ackerson T, et al. Guidelines for the Early Management of Patients With Acute Ischemic Stroke: 2019 Update to the 2018 Guidelines for the Early Management of Acute Ischemic Stroke: A Guideline for Healthcare Professionals From the American Heart Association/American Stroke Association. Stroke 2019;50:e344-e418.

21. Liu Z. Analysis of the effect of acute cerebral infarction with hyperhomocysteinemia by transfer-through-governor injection. Zhi Hui Jian Kang 2020;6:153-4,7.

22. Guan M. Effect of Du-mai three acupuncture mainly on CI and HS-CRP in patients with acute cerebral infarction: Zunyi 
Medical University; 2019.

23. Lai P. Effects of Electroacupuncture on immune and inflammatory responses in patients with acute cerebral infarction: Traditional Chinese Medicine University of Guangzhou 2019.

24. Xu S, Zeng Y, Bao H. Effects of tongdu acupuncture combined with Huanglian Wendan Decoction on Mir-132 and Mir-134 in peripheral blood of stroke patients. Zhongguo Xian Dai Yi Sheng 2019;57:137-40,44.

25. Hua H, Qian F, Zheng W. Effect of acupuncture and moxibustion combined with tongdu therapy on ischemic stroke and its influence on prognosis. Shanghai Zhen Jiu 2019;38:1087-92.

26. Song Z. Clinical observation on the therapeutic effect of tongyuan injection in treating acute ischemic stroke: Traditional Chinese Medicine University of Guangzhou; 2018.

27. Li Y. Study on the effect and curative effect of Tongdu Shenregulating acupuncture on ischemic penumbra in patients with acute ischemic stroke: Anhui University of Traditional Chinese Medicine; 2018.

28. Xu Q, Peng Y. Clinical effect observation and its influence on autophagy of acute cerebral infarction treated by "Tongdu acupuncture". Zhongguo Zhen Jiu 2018;38:457-61.

29. Liu X, Liu C. The clinical effect of transfer-directacupuncture on acute cerebral infarction complicated with hyperhomocysteine. China Health Care \& Nutrition 2017;27:88-9.

30. Luan H. Effect analysis of acupuncture combined with rehabilitation training in the treatment of hemiplegia after acute ischemic stroke. Zhong Xi Yi Jie He Yan Jiu 2017;9:4-5,11.

31. Zhang L, Wang Y, Liu W, et al. Effect of regulating acupuncture on upper limb muscle tone in patients with acute cerebral infarction. Hebei Zhong Yi Yao Xue Bao 2017;32:54-7.

Cite this article as: Shao TY, Ding MR, Ye ZX, Qian MX, Zhou X, Jin ZQ. Governor vessel acupuncture for acute ischemic stroke: a systematic review and meta-analysis. Ann Palliat Med 2021;10(7):7236-7246. doi: 10.21037/apm-21-691
32. Zhang L, Zhang G, Zhu L, et al. Clinical observation on the treatment of wind-phlegm-Stasis acute cerebral infarction by Tongdu-Tiaoshen acupuncture. Journal of Anhui University of Chinese Medicine 2017;36:59-62.

33. Cai J, Tang C, Zhu G. Clinical study on acupuncture Dumai point in the treatment of acute cerebral infarction. Ya Tai Chuan Tong Yi Yao 2016;12:116-7.

34. Ma X, Li J, Yan B, et al. Clinical study on the treatment of acute cerebral infarction complicated with hyperhomocysteine by transfer-general acupuncture. Journal of Shaanxi College of Traditional Chinese Medicine 2016;39:50-3,102.

35. LI X, Li K. Observation on the curative effect of acupuncture on acute ischemic stroke. Zhongguo Zhen Jiu 2009;29:5-6.

36. Zhang S, Chen D, Lai Z. Clinical observation of acupuncture Dumai point in the treatment of acute cerebral infarction. Journal of Changchun University of Traditional Chinese Medicine 2007;23:57-8.

37. Jin Z, Liang J, Wang J, et al. Delayed brain ischemia tolerance induced by electroacupuncture pretreatment is mediated via MCP-induced protein 1. J Neuroinflammation 2013;10:63.

38. Wang Q, Li X, Chen Y, et al. Activation of epsilon protein kinase C-mediated anti-apoptosis is involved in rapid tolerance induced by electroacupuncture pretreatment through cannabinoid receptor type 1 . Stroke 2011;42:389-96.

39. Tian H, Zhang H, Zhu J, et al. Acupuncture activates signal transduction pathways related to brain-tissue restoration after ischemic injury. Neural Regen Res 2012;7:1866-72.

40. Vase L, Baram S, Takakura N, et al. Can acupuncture treatment be double-blinded? An evaluation of double-blind acupuncture treatment of postoperative pain. PLoS One 2015;10:e0119612.

41. Zhang SH, Liu M, Asplund K, et al. Acupuncture for acute stroke. Cochrane Database Syst Rev 2005;(2):CD003317. 